\title{
Isentropic Analysis of Convective Motions
}

\author{
OLIVIER M. PAULUiS \\ Center for Atmosphere Ocean Science, Courant Institute of Mathematical Sciences, \\ New York University, New York, New York \\ AgNieszKa A. MrowiEC \\ Columbia University, and NASA Goddard Institute for Space Studies, New York, New York
}

(Manuscript received 19 July 2012, in final form 21 May 2013)

\begin{abstract}
This paper analyzes the convective mass transport by sorting air parcels in terms of their equivalent potential temperature to determine an isentropic streamfunction. By averaging the vertical mass flux at a constant value of the equivalent potential temperature, one can compute an isentropic mass transport that filters out reversible oscillatory motions such as gravity waves. This novel approach emphasizes the fact that the vertical energy and entropy transports by convection are due to the combination of ascending air parcels with high energy and entropy and subsiding air parcels with lower energy and entropy. Such conditional averaging can be extended to other dynamic and thermodynamic variables such as vertical velocity, temperature, or relative humidity to obtain a comprehensive description of convective motions. It is also shown how this approach can be used to determine the mean diabatic tendencies from the three-dimensional dynamic and thermodynamic fields.

A two-stream approximation that partitions the isentropic circulation into a mean updraft and a mean downdraft is also introduced. This offers a straightforward way to identify the mean properties of rising and subsiding air parcels. The results from the two-stream approximation are compared with two other definitions of the cloud mass flux. It is argued that the isentropic analysis offers a robust definition of the convective mass transport that is not tainted by the need to arbitrarily distinguish between convection and its environment, and that separates the irreversible convective overturning from oscillations associated with gravity waves.
\end{abstract}

\section{Introduction}

Atmospheric convection transports energy and water from Earth's surface to the free troposphere. The ascent of warm, moist air in saturated turbulent plumes is balanced by subsidence of drier and colder air that takes place in the environment or in convective or mesoscale downdrafts. Convective systems, however, rarely occur as simple overturning cells; rather, they are associated with a variety of turbulent motions over a wide range of scales. Any analysis of such flow is complex because individual air parcels undergo multiple dynamical and thermodynamical transformations. For simplicity, one

Corresponding author address: Olivier M. Pauluis, Center for Atmosphere Ocean Science, Courant Institute of Mathematical Sciences, New York University, 251 Mercer Street, New York, NY 10012.

E-mail: pauluis@cims.nyu.edu may want to separate irreversible ascent and mixing of air parcels from the gravity waves. However, such separation is not straightforward as convective plumes and gravity waves are often spatially and temporally collocated. The main purpose of this paper is to introduce a new technique to diagnose the convective overturning in numerical models.

The proposed approach takes advantage of the quasi conservation of entropy to isolate convective overturning from oscillatory motions. The concept of isentropic analysis (i.e., analyzing motions on surfaces of constant entropy) dates back to the early development of dynamical meteorology (Rossby 1937). As potential temperature and entropy are approximately conserved in the free troposphere, the motions of air parcels can be tracked on isentropic surfaces even when a lack of observations prevents determining their vertical displacement. This technique was initially focused on midlatitudes weather systems and led to the widespread use of

DOI: 10.1175/JAS-D-12-0205.1 
isentropic maps in synoptic meteorology. It was recognized early on that convective processes posed a specific challenge, as they involved a significant amount of mixing as well as latent heat release. To account for the latent heat release, several studies (Browning and Ludlam 1962; Ludlam 1963; Green et al. 1966) introduced the use of the wet-bulb potential temperature: an adiabatic invariant for reversible phase transition. Combining moist thermodynamics and isentropic analysis has been particularly influential in identifying the importance ascending moist air masses in the so-called moist conveyor belt (Harrold 1973). Isentropic coordinates have also been applied in studies of moist convection $\mathrm{Xu}$ and Emanuel (1989), tropical cyclogenesis (Schubert and Alworth 1987; Molinari et al. 1997), hurricane intensity (Molinari et al. 1995), and structure (Merrill and Velden 1996).

In this paper, we propose a systematic framework to analyze upward mass transport in moist convection by conditionally averaging it in terms of the equivalent potential temperature. The traditional isentropic analysis faces an important difficulty when applied to convective motions: moist isentropes exhibit an extremely complex geometry as they are continuously stretched and folded by turbulent convection. This complex geometry makes it virtually impossible to describe the instantaneous flow on a moist isentrope. However, we will show here that this limitation can be overcome by conditional averaging of the flow to obtain a mean isentropic circulation.

Our approach here is analogous to studies of the meridional circulation in isentropic coordinates. By averaging the meridional mass transport in terms of its potential temperature (Dutton 1976; Johnson 1989; Held and Schneider 1999) or of its equivalent potential temperature (Pauluis et al. 2008, 2010), one obtains a mean meridional circulation on dry or moist isentropes that is composed of a single equator-to-pole overturning cell in each hemisphere. This isentropic circulation is in sharp contrast to the Eulerian-mean circulation, obtained by averaging the velocity at constant pressure, which exhibits the well-known three-cell structure, with the Hadley cell in the tropics, Ferrel cell in the midlatitudes, and polar cell at high latitudes. The differences between the Eulerian and isentropic circulations offer an example of how conditional averaging in a turbulent flow can lead to new insights on how the turbulence is affecting parcel trajectories. The technique we develop here amounts to analyzing the isentropic circulation in the vertical direction and offers a systematic way to separate irreversible overturning by convective motions from reversible oscillations by gravity waves.

Section 2 introduces the isentropic averaging for the convective mass transport and defines the convective streamfunction. Our approach here is to compute the conditional average of vertical mass transport in terms of its equivalent potential temperature in a way that is similar to Kuang and Bretherton (2006). This mass transport can then be integrated to obtain a streamfunction, which offers a simple representation of the convective overturning. This methodology is used to analyze radiative-convective equilibrium simulations with the System for Atmospheric Modeling (SAM; Khairoutdinov and Randall 2003). In section 3, the isentropic averaging is generalized to assess the thermodynamic and dynamical properties of the air parcels. It can be used to define the probability of occurrence, mean vertical velocity, and mean thermodynamic properties such as water content and buoyancy of air parcels in terms of height and equivalent potential temperature. Thus, it is possible, for example, to isolate a population of intense, almost undiluted updrafts associated with peak vertical velocities of about $40 \mathrm{~m} \mathrm{~s}^{-1}$ in numerical simulations. Section 4 shows application of the isentropic analysis to determine the mean diabatic tendency from the convective streamfunction. Furthermore, entrainment rate can be determined empirically as functions of equivalent potential temperature and height. Section 5 introduces a two-stream decomposition of the convective motions based on the isentropic analysis. The convective circulation is partitioned between mean upward and mean downward flows of equal mass transport but different thermodynamic properties. These results are contrasted with two other definitions of the convective mass transport. It is shown here that, as a result of this comparison, the isentropic analysis leads to significantly lower value of the convective mass transport particularly in the upper troposphere.

\section{Isentropic streamfunction}

The isentropic averaging technique discussed below will be used to analyze a simulation of radiative-convective equilibrium performed with SAM, a cloud-resolving model developed by Khairoutdinov and Randall (2003). The model was integrated on a $216 \mathrm{~km} \times 216 \mathrm{~km} \times 28 \mathrm{~km}$ domain at 500-m horizontal resolution and stretched vertical grid with 64 gridpoints, with periodic boundary conditions in the horizontal directions. The lower boundary is an ocean surface at constant temperature of $301 \mathrm{~K}$, while a sponge layer is applied in the upper $8 \mathrm{~km}$ to prevent the reflection of gravity waves. The model uses a five species single-moment microphysics, an explicit radiative transfer, and was integrated for 100 days, with the last 60 days used for the time averaging. 
We refer to an isentropic slice as the intersection of an isentrope with a surface of constant height. We define the isentropic distribution of the variable $f$ as the integral of $f$ on a given isentropic slice:

$$
\langle f\rangle\left(z, \theta_{e 0}\right)=\frac{1}{P L_{x} L_{y}} \int_{0}^{P} \int_{0}^{L_{y}} \int_{0}^{L_{x}} f(x, y, z, t) \delta\left[\theta_{e 0}-\theta_{e}(x, y, z, t)\right] d x d y d t
$$

Here, $\theta_{e}$ is the equivalent potential temperature, $P$ is the time period over which the averaging is performed, and $L_{x}$ and $L_{y}$ are the horizontal extent of the domain. The integral in Eq. (1) involves a Dirac delta function $\delta\left[\theta_{e 0}-\theta_{e}(x, y, z, t)\right]$, which is approximated here by a function that is equal to $1 / \Delta \theta_{e}$ for $\theta_{e}$ between $\theta_{e 0}-0.5 \Delta \theta_{e}$ and $\theta_{e 0}+0.5 \Delta \theta_{e}$, and 0 elsewhere. In practice, Eq. (1) amounts to sorting the air parcels in terms of their equivalent potential temperature and to summing the quantity $f$ at each level in finite $\theta_{e}$ bins. The isentropic distribution defined by Eq. (1) is therefore a function of height and equivalent potential temperature. For simplicity of notation, the dependency on $\left(z, \theta_{e}\right)$ will not be explicitly indicated from now on, but it should be understood that all isentropic integrals (denoted by angle brackets) are functions of both $z$ and $\theta_{e}$. Note that the units of $\langle f\rangle$ are given by the units of $f$ per kelvin.

The equivalent potential temperature is defined following Emanuel (1994)'s Eq. (4.5.11):

$$
\theta_{e}=T\left(\frac{p_{0}}{p_{d}}\right)^{R_{d} / C_{p}} \exp \left(\frac{L_{v} r}{C_{p} T}\right) \mathcal{H}^{-R_{v} r / C_{p}}
$$

Here, $T$ is the temperature; $p_{0}$ is a reference pressure of $1000 \mathrm{hPa} ; p_{d}$ is the partial pressure of dry air; $R_{d}$ and $R_{v}$ are the ideal gas constants for dry air and water vapor, respectively; $C_{p}=C_{p d}+r_{T} C_{l}$ is the specific heat at constant pressure of moist air, with $C_{p d}$ and $C_{l}$ the specific heat of dry air and liquid water, respectively; $L_{v}$ is the latent heat of vaporization; $\mathcal{H}$ is the relative humidity; and $r$ and $r_{T}$ are the mixing ratios for water vapor and total water. In our study, we included cloud water and ice as part of the total water content but excluded precipitation from the computation of $\theta_{e}$. Alternative choices for a variable used to define isentropic surfaces are addressed in the discussion section at the end of the paper.

The isentropic distribution of the vertical mass flux $\langle\rho w\rangle$, where $\rho$ is the mass per unit volume and $w$ is the vertical velocity, in the radiative-convective equilibrium simulation is shown in Fig. 1a. The solid black line shows the horizontal-mean profile of equivalent potential temperature $\bar{\theta}_{e}(z)$. The units of $\langle\rho w\rangle\left(\mathrm{kg} \mathrm{m}^{-2} \mathrm{~s}^{-1} \mathrm{~K}^{-1}\right)$ correspond to a vertical mass flux per unit area and per unit of equivalent potential temperature. The quantity $\langle\rho w\rangle \delta \theta_{e}$ corresponds to the net vertical mass flux of air parcels at level $z$ with an equivalent potential temperature between $\theta_{e}$ and $\theta_{e}+\delta \theta_{e}$.

The isentropic distribution of the vertical mass flux can be used to define an isentropic streamfunction as

$$
\Psi\left(z, \theta_{e}\right)=\int_{-\infty}^{\theta_{e}}\langle\rho w\rangle\left(z, \theta_{e}^{\prime}\right) d \theta_{e}^{\prime}
$$

From a physical point of view, the streamfunction $\Psi\left(z, \theta_{e}\right)$ can be interpreted as the net vertical mass flow per unit area at level $z$ of all air parcels with an equivalent potential temperature less than $\theta_{e}$.

The isentropic streamfunction is shown in Fig. 1b. Given the definition (3), the streamfunction at a given height first decreases with $\theta_{e}$, as air parcels with low equivalent temperature are on average subsiding, until it reaches its minimum value for the value of $\theta_{e}$ at which $\langle\rho w\rangle$ changes sign. That the streamfunction is negative through most of the troposphere indicates that rising air parcels have, on average, a higher equivalent potential temperature than the subsiding air, and thus corresponds to an upward transport of $\theta_{e}$. The absolute minimum of the streamfunction is located near the surface and is associated with mixing within the subcloud layer. The magnitude of the streamfunction decreases sharply above $1 \mathrm{~km}$, and then decreases more gradually with height all the way to the tropopause, corresponding to a continuous detrainment of air from the updrafts. The streamfunction changes sign at about $12 \mathrm{~km}$, pointing to the presence of convective overshoots associated with a weak downward entropy and energy transport as rising air mixes with air parcels with higher potential temperature before subsiding.

In section 4 , we show that that the vertical derivative of the streamfunction is proportional to minus the mean diabatic tendency of air parcels and that the mean trajectory of air parcels in $z-\theta_{e}$ coordinates is along the streamline. As with any turbulent flow, one should be aware that individual parcel trajectories may differ significantly from the mean velocity in the flow. Ascending air parcels originating from the lowest atmospheric layer have high values of $\theta_{e}$, up to $355 \mathrm{~K}$. The equivalent potential temperature of the ascending air, however, drops rapidly with height, indicating entrainment of drier air in 

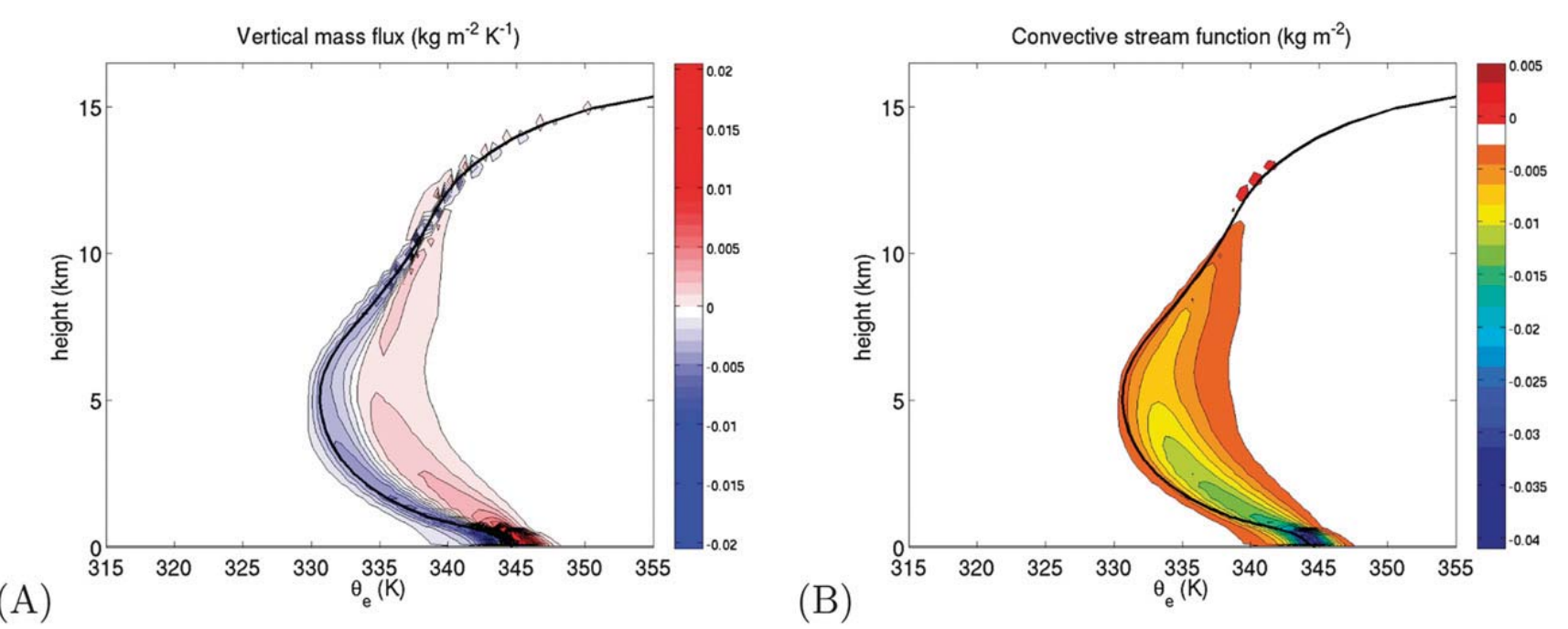

FIG. 1. (a) Isentropic distribution of vertical mass flux $\langle\rho w\rangle\left(\mathrm{kg} \mathrm{m}^{-2} \mathrm{~s}^{-1} \mathrm{~K}^{-1}\right)$ in the radiative-convective equilibrium simulations.

(b) Isentropic streamfunction $\Psi\left(z, \theta_{e}\right)\left(\mathrm{kg} \mathrm{m}^{-2} \mathrm{~s}^{-1}\right)$. The solid line shows the mean profile of equivalent potential temperature $\bar{\theta}_{e}(z)$.

the updrafts. Above $4-5 \mathrm{~km}$, the streamlines become almost vertical, corresponding to a vanishing mean diabatic tendency in the updraft, which would indicate that the role of entrainment is limited above the freezing level. As adiabatic freezing or sublimation can lead to an increase in the equivalent potential temperature, the vertical streamlines above the freezing level might actually be the results of the compensation between freezing and entrainment.

The bulk of the descending motion occurs at equivalent potential temperature close to the horizontalmean atmospheric state. Furthermore, the streamlines are closely aligned with $\bar{\theta}_{e}(z)$. The subsiding part of the circulation corresponds to a slow subsidence of air parcels whose equivalent potential temperature remains close to $\bar{\theta}_{e}(z)$. The minimum value of $\bar{\theta}_{e}(z)$ is $330 \mathrm{~K}$ and is located at $5 \mathrm{~km}$, near the freezing level. Above this minimum, the equivalent potential temperature of the subsiding air decreases as a direct consequence of radiative cooling. In contrast, below the minimum of $\bar{\theta}_{e}$ the equivalent potential temperature of subsiding air parcels gradually increases as they approach the surface. While radiative cooling is still present, the increase of $\theta_{e}$ is directly tied to the mixing between subsiding environmental air and detrained cloudy air with a higher value of $\theta_{e}$. Mixing between rising clouds and the surrounding air is associated with an exchange of latent heat from the former to the latter. This process can be directly diagnosed by the tilt of the streamfunction in the lower troposphere, which is reflected in both a gradual reduction of the equivalent potential temperature of the ascending air and a comparable increase of the equivalent potential temperature in subsiding air.

\section{Isentropic averaging of convective motions}

The isentropic integral (1) is not limited to vertical mass flux. The same formalism can be applied to any variable of interest to obtain a more detailed analysis of the typical properties of the air parcels involved in convective motions. The probability density function for a parcel with equivalent potential temperature $\theta_{e}$ at level $z$ can be estimated as

$$
\begin{aligned}
& \langle\text { Eq. }(1)\rangle\left(z, \theta_{e}\right) \\
& =\frac{1}{P L_{x} L_{y}} \int_{0}^{P} \int_{0}^{L_{y}} \int_{0}^{L_{x}} \delta\left[\theta_{e 0}-\theta_{e}(x, y, z, t)\right] d x d y d t .
\end{aligned}
$$

Indeed, the quantity $\langle$ Eq. (1) $\rangle\left(z, \theta_{e 0}\right) \delta \theta_{e}$ is equal to the fractional area covered by all parcels with equivalent potential temperature between $\theta_{e 0}$ and $\theta_{e 0}+\delta \theta_{e}$ at level $z$.

The logarithm of the probability density function is shown in Fig. 2a. The rising air parcels observed in Fig. 1 are associated with very low probability. For example, at an altitude of $5 \mathrm{~km}$, the ascending air has an equivalent potential temperature greater than $333 \mathrm{~K}$, and is associated with a probability density less than $0.003 \mathrm{~K}^{-1}$. All together the air parcels with $\theta_{e}>333 \mathrm{~K}$ account for less than $0.7 \%$ of all air parcels at that level. In contrast, subsiding air occupies most of the domain. As noted earlier, the equivalent potential temperature of subsiding air parcels is close to $\bar{\theta}_{e}(z)$, which also corresponds to the maximum of probability density function. For example, at an altitude of $5 \mathrm{~km}$, the mean subsidence in Fig. 2a occurs for $\theta_{e}<333 \mathrm{~K}$ and accounts for more than $99 \%$ of the air at that level. Subsiding air corresponds to the bulk of the atmosphere at any level, while rising thermals are rare occurrences. 

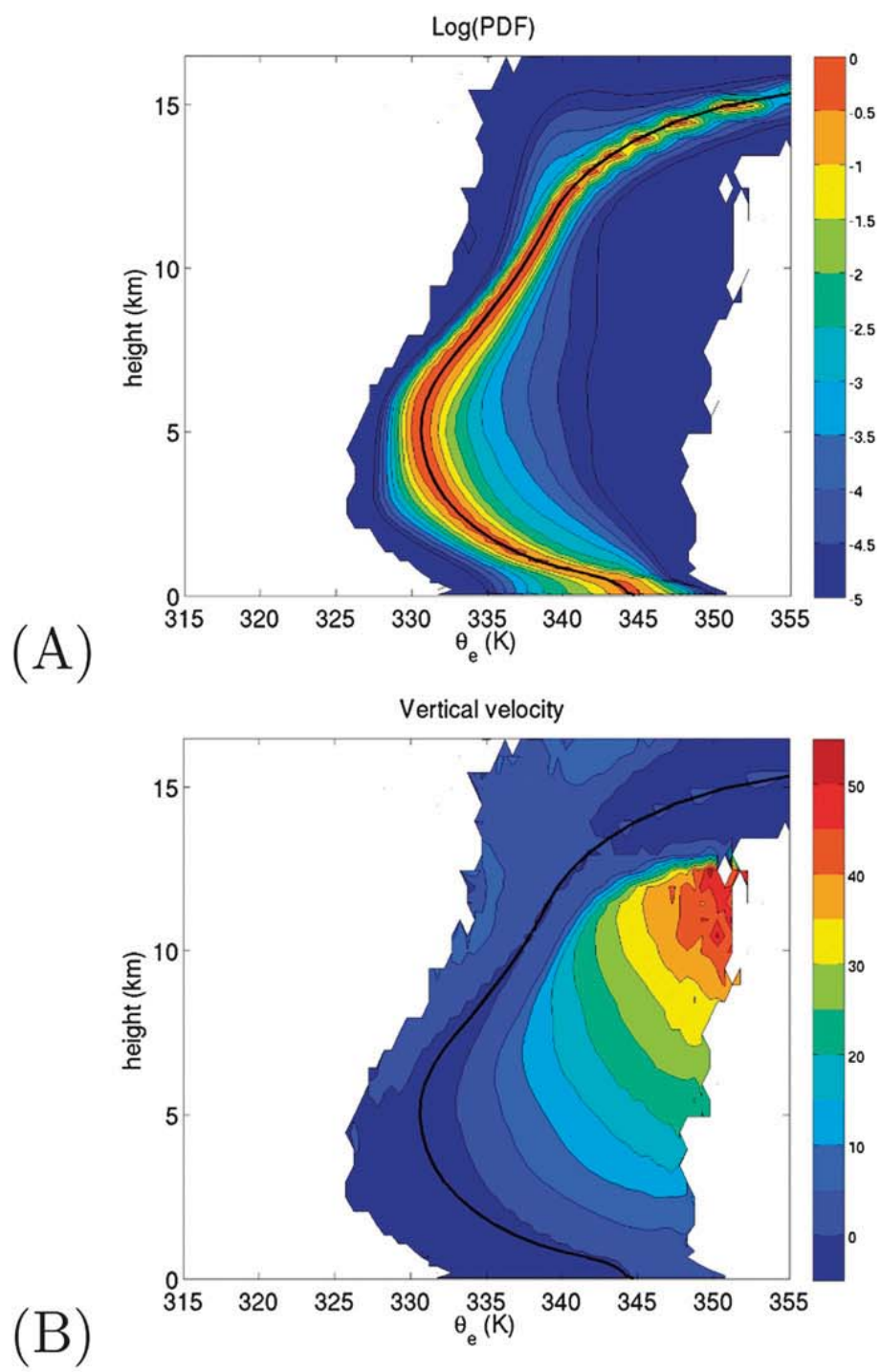

(B)

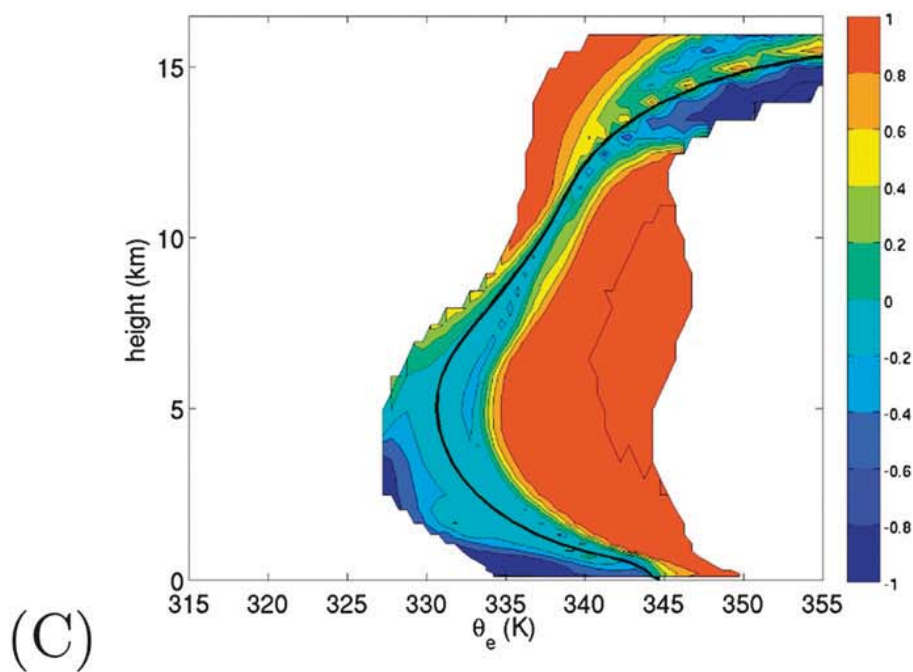

FIG. 2. (a) Logarithm (in base 10) of the probability density function $\langle$ Eq. (1) $\rangle\left(z, \theta_{e}\right)$. (b) Isentropic-mean vertical velocity $\tilde{w}\left(z, \theta_{e}\right)\left(\mathrm{m} \mathrm{s}^{-1}\right)$. (c) Directionality of the vertical mass flux. See text for definitions. 
The tails of the probability distribution also reveal some interesting aspects of convection. First, we note that the tail of the distribution at high $\theta_{e}$ closely follows a line of constant value of $\theta_{e}$. This indicates that undiluted air parcels from the boundary layer can be found through the entire troposphere. They are, however, very scarce (with a probability density less than $10^{-4} \mathrm{~K}^{-1}$ ) and do not contribute significantly to the total vertical mass transport. In contrast, the tail of the distribution for low values of $\theta_{e}$ does not exhibit any similar heightindependent cutoff. Indeed, values of $\theta_{e}$ of $330 \mathrm{~K}$ or less are found mostly within the midtroposphere between 3 and $7 \mathrm{~km}$. While one would not expect an air parcel with such low value of $\theta_{e}$ to rise in the upper troposphere where it would be negatively buoyant, one could imagine finding such a parcel near the surface if it had been brought there by a convective downdraft. As the surface distribution of equivalent potential temperature exhibits a cutoff at about $336 \mathrm{~K}$ (the fractional area of all air parcels with $\theta_{e}<336 \mathrm{~K}$ at the surface is less than $10^{-5}$ ), we can exclude the presence of strong convective downdraft that would bring undiluted air from the midtroposphere down to the surface. In fact, $\bar{\theta}_{e}(z)$ reaches a value of $336 \mathrm{~K}$ at approximately $1300 \mathrm{~m}$, which implies that undiluted downdrafts that reach the surface must originate from within the planetary boundary layer in our simulation.

The isentropic distributions for $\langle\rho w\rangle$ and for density $\langle\rho\rangle$ can be combined to define a horizontal- and isentropic-mean vertical velocity $\tilde{w}\left(\theta_{e}, z\right)$ for air parcels with a given value of $\theta_{e}$ at level $z$ :

$$
\tilde{w}\left(z, \theta_{e}\right)=\frac{\langle\rho w\rangle\left(z, \theta_{e}\right)}{\langle\rho\rangle\left(z, \theta_{e}\right)} .
$$

For simplicity, we refer to $\tilde{w}$ as the isentropic-mean vertical velocity for all air parcels for a given value of $\theta_{e}$ and $z$. Figure $2 \mathrm{~b}$ shows the isentropic-mean vertical velocity in our simulation. It exhibits a marked asymmetry between fast upward ascent at high $\theta_{e}$ and very slow subsidence at value of $\theta_{e}$ corresponding to the horizontalmean value, and is in good agreement with the conceptual model of Bjerkness (1938). The high values of $\tilde{w}$ (up to $50 \mathrm{~m} \mathrm{~s}^{-1}$ ) observed in the upper troposphere at high $\theta_{e}$ correspond to very strong, rare, weakly diluted or undiluted updrafts. The abrupt decrease of high $\tilde{w}$ at $12 \mathrm{~km}$ does not necessarily indicate that these strong updrafts disappear at that level. Rather, as $\tilde{w}$ is the average vertical velocity for all parcels at a given level and equivalent potential temperature [Eq. (5)], the value of $\tilde{w}$ decreases near the mean profile as fast rising parcels have the same potential temperature as a much larger number of slow-moving parcels in the environment.
Analysis of higher moments of the distribution could be used to further investigate the overshoot of these strong updrafts above their level of neutral buoyancy. Interestingly, while the isentropic analysis did detect strong updrafts associated with high values of $\theta_{e}$, there is little indication of strong downward motions at low values of $\theta_{e}$ that would correspond to strong convective downdrafts.

The distribution of $\langle\rho w\rangle$ is obtained by averaging over a large number of air parcels. The net vertical mass flux is often the result of a compensation between rising and subsiding motions. To quantify the amount of compensation, we introduce the net upward and downward velocities ( $w^{+}$and $w^{-}$, respectively) as

$$
\begin{aligned}
& w^{+}=\max (w, 0) \quad \text { and } \\
& w^{-}=\min (w, 0) .
\end{aligned}
$$

We then compute the corresponding isentropic distribution $\left(\left\langle\rho w^{+}\right\rangle\right.$and $\left.\left\langle\rho w^{-}\right\rangle\right)$and define the directionality of the mass transport at each $z$ and $\theta_{e}$ as

$$
A\left(z, \theta_{e}\right)=\frac{\langle\rho w\rangle\left(z, \theta_{e}\right)}{\max \left[\left\langle\rho w^{+}\right\rangle\left(z, \theta_{e}\right),-\left\langle\rho w^{-}\right\rangle\left(z, \theta_{e}\right)\right]} .
$$

A value of $A\left(z, \theta_{e}\right)$ close to unity indicates that air parcels at $z$ and $\theta_{e}$ are almost uniformly moving upward. Similarly, for a value of $A$ close to -1, air parcels are almost all moving downward. In contrast, when $A$ is close to zero, the net $\langle\rho w\rangle$ is the result of large compensation between ascending and subsiding motions. Figure $2 \mathrm{c}$ shows the directionality computed for our simulations. The directionally is close to one in the portions of the $z-\theta_{e}$ space associated with the mean ascent (i.e., for roughly $\theta_{e}>335 \mathrm{~K}$ and $2<z<10 \mathrm{~km}$ ). This indicates that air parcels with high $\theta_{e}$ are systematically ascending. In contrast, for values of $z-\theta_{e}$ near $\bar{\theta}_{e}(z)$, the directionality is almost zero. This corresponds to the bulk of the air at any level, which is associated with a net downward motion. Such low value of the directionality indicates that, as air outside of the convective updrafts is continuously oscillating because of the influence of propagating gravity waves, the subsidence is the residual transport that remains after a large compensation between the upward $\left(\left\langle\rho w^{+}\right\rangle\right)$and downward $\left(\left\langle\rho w^{-}\right\rangle\right)$contributions.

The averaging procedure used to define the isentropicmean vertical velocity can be applied to any variable. We define the mass-weighted isentropic mean for variable $f$ as

$$
\tilde{f}\left(z, \theta_{e}\right)=\frac{\langle\rho f\rangle}{\langle\rho\rangle} .
$$


(A)

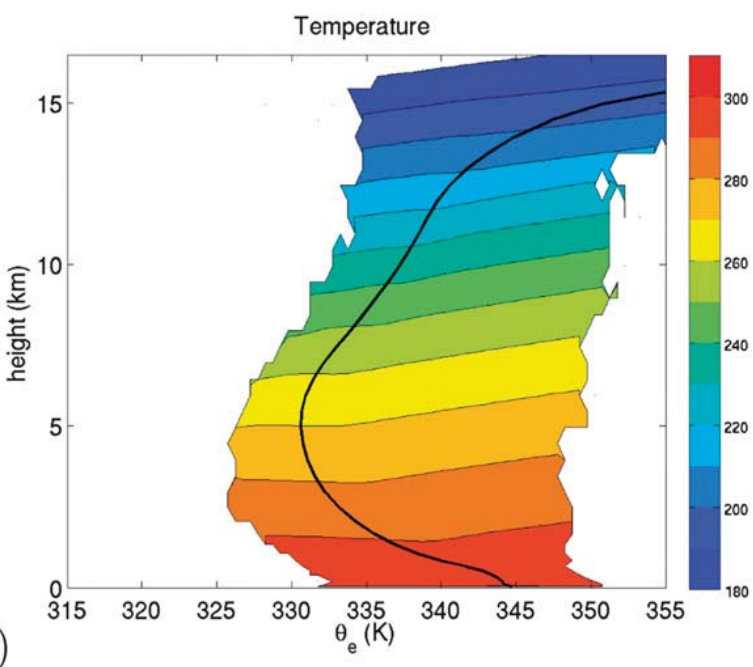

(C)

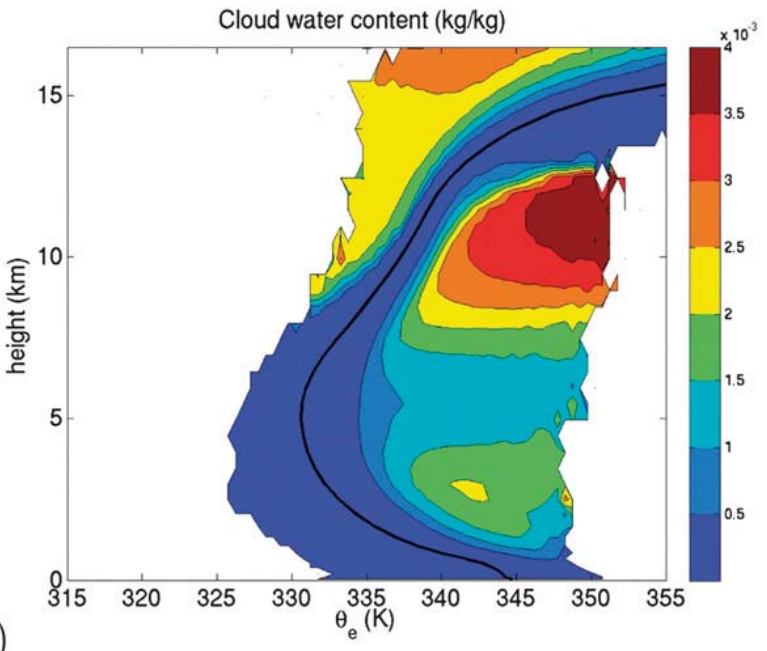

(B)
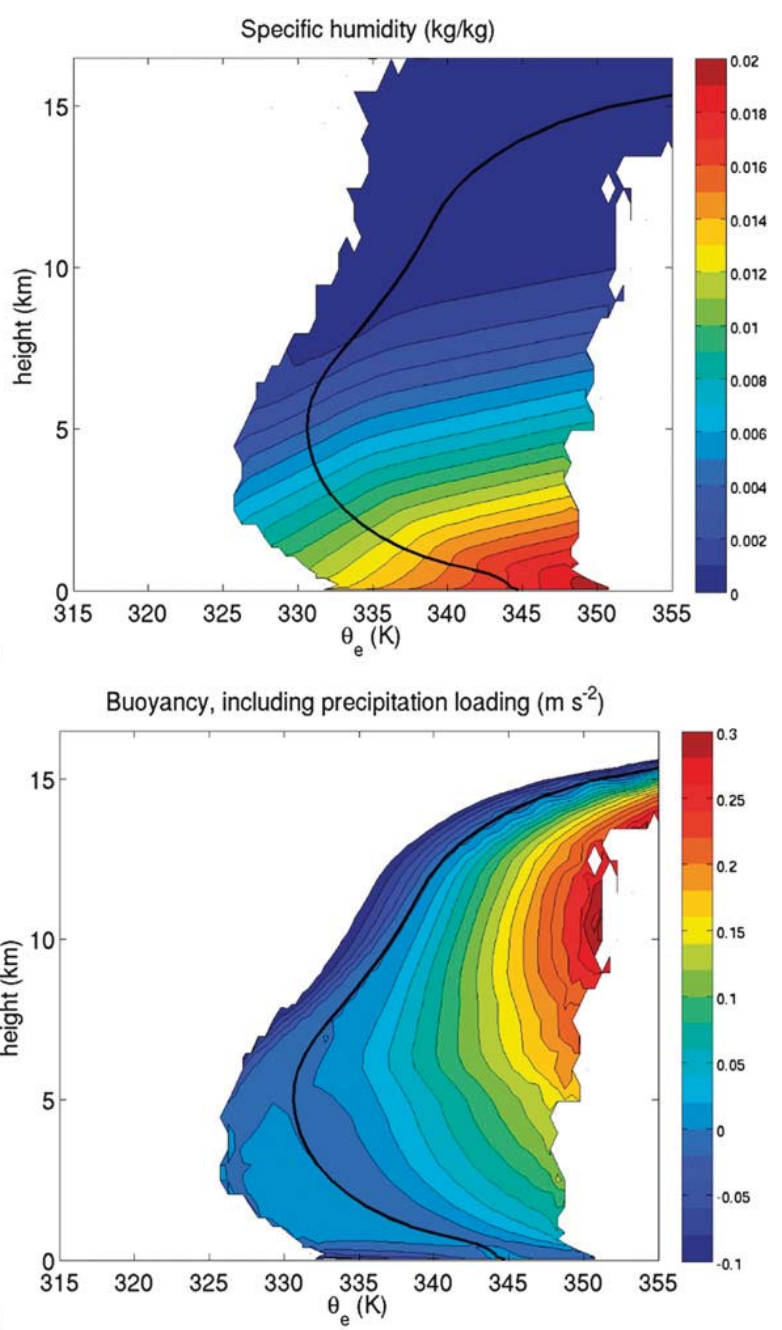

FIG. 3. (a) Isentropic-mean temperature $\tilde{T}\left(z, \theta_{e}\right)(\mathrm{K})$. (b) Isentropic-mean specific humidity $\tilde{q}\left(z, \theta_{e}\right)\left(\mathrm{kg} \mathrm{kg}^{-1}\right)$. (c) Isentropic-mean cloud water concentration $\tilde{q}_{c}\left(z, \theta_{e}\right)\left(\mathrm{kg} \mathrm{kg}^{-1}\right)$. (d) Isentropic-mean buoyancy $\tilde{B}\left(z, \theta_{e}\right)\left(\mathrm{m} \mathrm{s}^{-2}\right)$.

This formulation allows us to define the thermodynamic properties of the air parcels as function of their height and equivalent potential temperature. Figure 3 shows the mean value of the temperature $\tilde{T}$ (Fig. 3a), specific humidity $\tilde{q}$ (Fig. $3 \mathrm{~b}$ ), condensed water content $\tilde{q}_{l}+\tilde{q}_{i}$ (Fig. 3c), and buoyancy $\tilde{B}$ (Fig. 3d). The temperature decreases with height until its minimum near the tropopause. The coldest temperatures are associated with overshoots from the deep convective towers at around $15 \mathrm{~km}$. There is a weak inflection line in the temperature distribution (Fig. 3a). A similar inflection line is also present in the humidity distribution (Fig. 3b) for the same values of $z$ and $\theta_{e}$. The inflection marks the separation between unsaturated parcels to the left of the line and saturated parcels to the right. As discussed in Stevens (2005) and Pauluis (2008), the saturation of air parcels is characterized by a discontinuity in the equation of state. This means, for example, that the partial derivative

$$
\left(\frac{\partial T}{\partial \theta_{e}}\right)_{p, q_{T}}
$$

has a different value depending on whether a parcel is saturated or not. It is confirmed by the distribution of $\tilde{q}_{l}+\tilde{q}_{i}$ (Fig. 3c), which shows that the inflection lines in the distributions of $\tilde{T}$ and $\tilde{q}$ indeed correspond to appearance of condensate. ${ }^{1}$

The distribution of buoyancy is shown in Fig. 3d. Typical values for the buoyancy in rising air are on the order of $0.1 \mathrm{~m} \mathrm{~s}^{-2}$, while the buoyancy in the subsiding air parcels is significantly lower. This is consistent with

\footnotetext{
${ }^{1}$ The quantities $\tilde{T}$ and $\tilde{q}_{v}$ are obtained by averaging over both saturated and unsaturated parcels, which can smooth the discontinuity in the partial derivatives. The fact that one is apparent in Figs. $3 \mathrm{a}$ and $3 \mathrm{~b}$ indicates that saturated and unsaturated air parcels can be fairly well separated by their value of $\theta_{e}$ at a given level.
} 


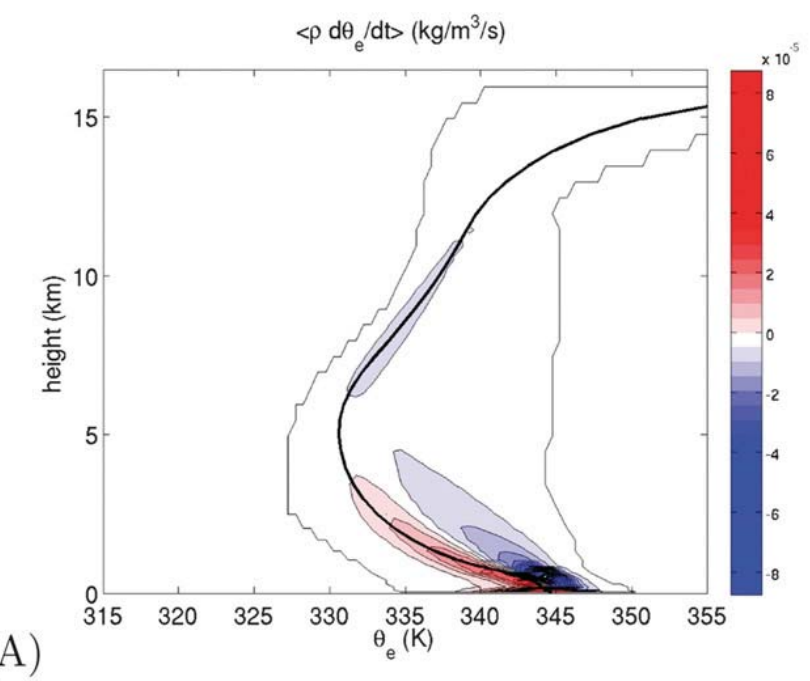

(B)
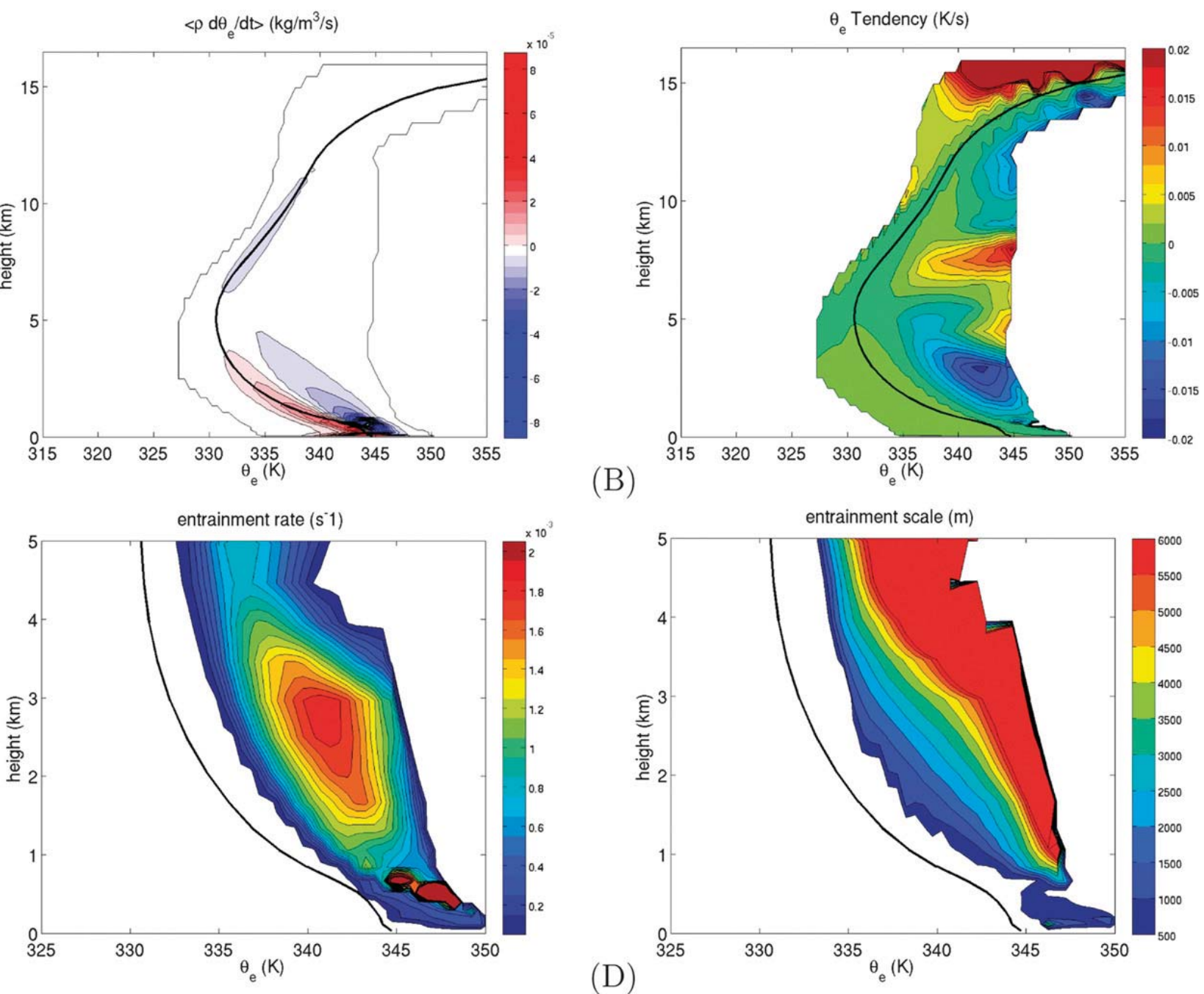

FIG. 4. (a) Isentropic distribution of diabatic heating $\left\langle\rho \dot{\theta}_{e}\right\rangle$. (b) Isentropic-mean diabatic heating tendency $\tilde{\dot{\theta}}_{e}\left(\mathrm{~K} \mathrm{~s}^{-1}\right)$. (c) Entrainment rate $\left(\mathrm{s}^{-1}\right)$ from Eq. (12). (d) Entrainment scale height (m) from Eq. (13).

the asymmetry between strong updraft and weak subsidence observed for the $\tilde{w}$. Note the gradual increase of the buoyancy with equivalent potential temperature, which is in agreement with the relationship between entropy and buoyancy discussed in Pauluis (2008). There is also a marked increase in the buoyancy of the air parcels at about $5 \mathrm{~km}$, which is most likely associated with the freezing of the liquid water in the convective updrafts.

\section{Diabatic tendency}

For a statistically steady system, the continuity equation can be written as

$$
\frac{\partial}{\partial z}\langle\rho w\rangle+\frac{\partial}{\partial \theta_{e}}\left\langle\rho \dot{\theta}_{e}\right\rangle=0
$$

This equation arises from the mass conservation expressed in $\theta_{e}$ and $z$ coordinates and is derived in the appendix.
The second term on the left-hand side corresponds to mass-weighted average of the diabatic tendency $\dot{\theta}_{e}$. Equation (9) combined with the definition of the streamfunction (3) makes it possible to express the diabatic tendency in terms of the vertical derivative of the streamfunction:

$$
\left\langle\rho \dot{\theta}_{e}\right\rangle=-\frac{\partial \Psi}{\partial z} .
$$

The mass-weighted diabatic tendency $\left\langle\rho \dot{\theta}_{e}\right\rangle$ is shown in Fig. 4a. Large heating rates are found near the surface corresponding to the surface latent and sensible heat fluxes. In the lower troposphere, a dipole of positive tendency at lower $\theta_{e}$ and negative tendency at higher $\theta_{e}$ is a result of diffusion of water vapor from moist updrafts to the drier environment. The upper troposphere is dominated by the negative tendencies associated with radiative cooling. 
Alternatively, the isentropic-mean diabatic tendency can also be expressed as

$$
\tilde{\dot{\theta}}_{e}=\frac{\left\langle\rho \dot{\theta}_{e}\right\rangle}{\langle\rho\rangle}
$$

and is shown in Fig. 4b. Large negative values of the diabatic tendency (on the order of $1000 \mathrm{~K} \mathrm{day}^{-1}$ ) are associated with the diffusion of water vapor out of cloudy air parcels. This contrasts with a much slower rate of increase in $\theta_{e}$ associated with the moistening of dry air, as the water vapor flux is diffused into a much larger mass of environmental air.

The distribution of diabatic heating in the lower troposphere can be explained through a simple model that assumes that ascending air parcels entrain air from the environment. If one neglects the effects of radiative cooling, the potential temperature tendency in the updrafts can be written as

$$
\frac{d \theta_{e}}{d t}=\frac{\bar{\theta}_{e}-\theta_{e}}{\tau_{e}}
$$

where $\tau_{e}$ corresponds to the time it takes to mix environmental air (assumed here to have the same properties as the horizontal-mean profile) into an updraft. This time scale can be estimated based on the isentropic analysis as

$$
\tau_{e}\left(z, \theta_{e}\right)=\frac{\bar{\theta}_{e}-\theta_{e}}{\tilde{\theta}_{e}}
$$

The product of the entrainment time scale and the vertical velocity can then be expressed in terms of an entrainment scale height:

$$
\lambda_{e}\left(z, \theta_{e}\right)=\frac{\tilde{w}\left(\bar{\theta}_{e}-\theta_{e}\right)}{\tilde{\theta}_{e}} .
$$

The entrainment rate and scale height for the radiativeconvective equilibrium simulations are shown in Figs. $4 \mathrm{c}$ and 4d. Kuang and Bretherton (2006) use a similar Eq. (11) to assess the effects of entrainment on parcel trajectories. However, in their approach, one must first assume a given value of the entrainment rate to determine the value of the equivalent potential temperature following an air parcel at various heights. In contrast, here we use the results from the isentropic analysis to determine the mean rate of change of the equivalent potential temperature, which is then used to determine effective entrainment rate. From a geometric point of view, the quantity

$$
\frac{\tilde{w}}{\tilde{\dot{\theta}}_{e}}=\frac{\langle\rho w\rangle}{\left\langle\rho \dot{\theta}_{e}\right\rangle}=\frac{\partial \Psi / \partial \theta_{e}}{\partial \Psi / \partial z}
$$

is the slope of a streamline determined form the isentropic analysis in Fig. 1b. The entrainment scale height is obtained by normalizing this slope by the distance to $\overline{\theta_{e}}(z)$. When the streamlines are almost vertical, the entrainment-scale height is large, indicating little or no entrainment. Conversely, when the streamlines are almost horizontal, the entrainment scale height is small, corresponding to strongly entraining plumes. It should also be stressed that Eq. (11) assumes that the change of $\theta_{e}$ is solely due to mixing between rising air and an environment, thus neglecting the effect of radiation and freezing. The latter can result in a positive tendency $\dot{\theta}_{e}$ in the updraft, in which case Eq. (12) yields a negative entrainment rate. The definition of the local entrainment rate in Eq. (12) should not be applied above the freezing point of water.

These diagnostics for entrainment shown in Figs. 4c and $4 \mathrm{~d}$ reveal a very large amount of mixing within the subcloud layer, which is associated with a large number of the shallow overturning eddies that do not rise above $1 \mathrm{~km}$. The mixing height there can be on the order of $500 \mathrm{~m}$ or less, which corresponds to the model resolution. In contrast, in the free troposphere, the distribution of mixing-scale height varies from relatively short $(1 \mathrm{~km}$ or less) for parcels founds near the mean profile to value of several kilometers at large value of $\theta_{e}$ typical of the subcloud layer. Updrafts with high values of $\theta_{e}$ correspond to almost undiluted air parcels but are quite scarce, while the bulk of the ascending air is associated with weaker but more entraining updrafts. The fact that Eq. (13) yields very different entrainment scale for different values of $\theta_{e}$ indicates that a bulk entrainment rate obtained from plume models [see de Rooy et al. (2013) for a review] only characterizes mixing in an average sense and does not necessarily apply to individual parcels.

\section{Mass flux and entrainment from two-stream approximation}

The isentropic analysis discussed in the previous section offers an efficient way to characterize the thermodynamic properties of convective updrafts and downdrafts. A two-stream approximation is introduced here to synthesize this information. The convective overturning is divided into a mean ascent and a mean descent based on the convective streamfunction. First, we define the upward and downward mass transports $\left(M^{+}\right.$and $M^{-}$, respectively): 
(A)

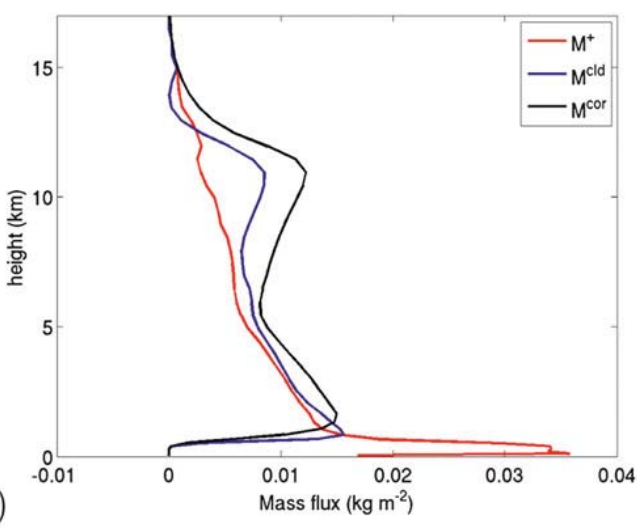

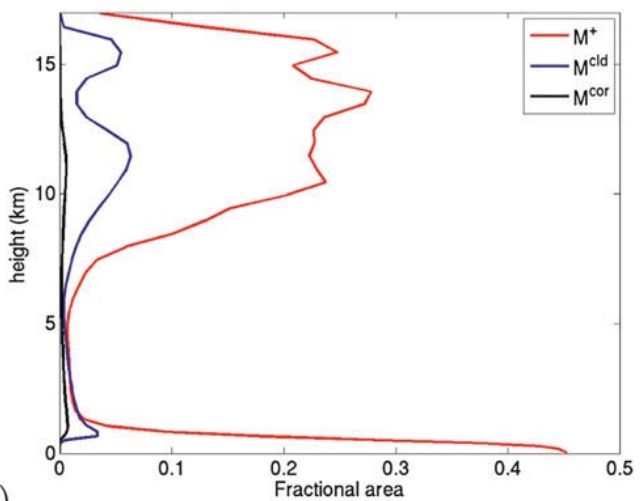

FIG. 5. Comparison between the two-stream approximation $M^{+}$(red), cloud mass flux $M_{\text {cld }}$ (blue), and convective core $M_{\text {cor }}$ (black): (a) mass flux and (b) fractional area.

$$
\begin{aligned}
& M^{+}(z)=\int_{-\infty}^{\infty}\langle\rho w\rangle H(\langle\rho w\rangle) d \theta_{e} \text { and } \\
& M^{-}(z)=\int_{-\infty}^{\infty}\langle\rho w\rangle H(-\langle\rho w\rangle) d \theta_{e}
\end{aligned}
$$

where $H$ is a Heaviside step function. As the net mass transport vanishes, mass transports in the mean ascent and descent cancel each other out in the absence of mean vertical motion: $M^{+}+M^{-}=0$.

The mass flux obtained from the isentropic analyses can be compared to other definitions of the convective mass transport. Two common measures of the convective mass transport are the vertical mass flux in cloudy air $M_{\text {cld }}$ and the vertical mass flux in convective cores $M_{\text {cor }}$, which are defined respectively as

$$
\begin{aligned}
& M_{\mathrm{cld}}(z)=\frac{1}{P L_{x} L_{y}} \int_{o}^{P} \int_{0}^{L_{y}} \int_{0}^{L_{x}} w(x, y, z, t) H\left(q_{c}-\epsilon\right) d x d y d t \text { and } \\
& M_{\mathrm{cor}}(z)=\frac{1}{P L_{x} L_{y}} \int_{o}^{P} \int_{0}^{L_{y}} \int_{0}^{L_{x}} w(x, y, z, t) H\left(q_{c}-\epsilon\right) H(w-1) d x d y d t,
\end{aligned}
$$

where $q_{c}$ is a mass mixing ratio of cloud water, $\epsilon=$ $10^{-10} \mathrm{~g} \mathrm{~kg}^{-1}$ is a small threshold used for determining the presence of cloud water, and cores are defined with a threshold on vertical velocity: $|w| \geq 1 \mathrm{~m} \mathrm{~s}^{-1}$.

The vertical profile for $M^{+}$from the two-stream approximation is contrasted with $M_{\text {cld }}$ and $M_{\text {cor }}$ in Fig. 5a, while the fractional area covered by clouds, convective clouds and the isentropic ascent are shown in Fig. 5b. Below the condensation level, both $M_{\text {cld }}$ and $M_{\text {cor }}$ vanish by definition, but $M^{+}$is large, as the streamfunction captures the convective motions in the subcloud layer. The isentropic mass flux drops sharply at the cloud base, and the three mass fluxes have similar values within the lower troposphere between 1 and $5 \mathrm{~km}$. The fact that the mass flux in convective cores is slightly larger than the other two indicates that some of the air that rises within the convective cores must fall back while remaining saturated. The fractional area covered by the convective cores is also significantly smaller than the fractional areas associated with the isentropic ascent and with clouds. The three fractional areas are, however, small, on the order of $1 \%-2 \%$ through the lower troposphere. The similarities in the mass fluxes and in the fractional areas in the lower troposphere further confirm that the isentropic ascent occurs within saturated updraft.

In the upper troposphere, from 5 to $12 \mathrm{~km}$, the mass fluxes and fractional areas differ significantly depending on their definition. Both $M_{\text {cld }}$ and $M_{\text {cld }}$ exhibit a secondary maximum in the upper troposphere at about $12 \mathrm{~km}$. In contrast, $M^{+}$decreases monotonically. The fractional areas associated with isentropic ascent and with clouds increase greatly, whereas the fractional area of convective cores remains very small. To understand these differences, we need to consider the nature of convection in the upper troposphere: saturated updrafts rise to their level of neutral buoyancy, overshoot it then settle down, forming extensive anvil clouds that are slowly dissipated 
through precipitation and mixing. The convective cores are defined as cloudy air that moves up at a velocity larger than $1 \mathrm{~m} \mathrm{~s}^{-1}$. This velocity threshold implies that $M_{\text {cor }}$ only captures the ascent during the overshoot, but not the downward motion that follows as the air moves back down toward its level of neutral buoyancy. Furthermore, as air rises in a stratified environment, it generates internal gravity waves characterized by significant fluctuations of the vertical velocity away from the updraft itself. If these fluctuations exceed the threshold of $1 \mathrm{~m} \mathrm{~s}^{-1}$ within a cloud, then such gravity wave would result in a positive contribution to $M_{\text {cor }}$ even in the absence of overturning. Similarly, if a gravity wave propagates through a dissipating cloud, it will be associated with a net upward contribution to $M_{\text {cld }}$ as Eq. (16a) registers the gradual increase of the condensation level. The increase in $M_{\text {cld }}$ and in $M_{\text {cor }}$ reflects interactions between clouds and gravity waves and should not be interpreted as an increase in mass flux due to entrainment. This problem arises directly from the difficulty in separating gravity waves from clouds or convective cores in the upper troposphere.

The isentropic mass flux exhibits a continuous decrease throughout the upper troposphere without any secondary maximum. An important aspect of the isentropic mass flux lies in that it is mostly unaffected by propagating gravity waves. Indeed, in the linear theory, the vertical velocity field of a gravity wave is out of phase with the equivalent potential temperature perturbation and thus does not result in any net vertical displacement of the air parcel over a wave period. Such result can be further extended under the generalized Lagrangianmean framework to show that steady, nondissipative waves do not produce any mean Lagrangian displacement (Andrews and McIntyre 1978; McIntyre 1980; Andrews et al. 1987). Gravity waves will only affect the isentropic mass transport if they are breaking or dampened. Such breaking can be noticed in Fig. 1a with the dipoles of ascent/subsidence for $\theta_{e}>340 \mathrm{~K}$ and $z>$ $12 \mathrm{~km}$, but the corresponding mass transport is small. The area associated with the isentropic ascent increases dramatically. This area is defined as the total area of all air parcels whose equivalent potential temperature is such that $\langle\rho w\rangle\left(z, \theta_{e}\right)$ is positive. Given that $\langle\rho w\rangle$ itself is an average, this area includes some parcels that may be moving down. The increase in the area covered by the isentropic ascent reflects the fact that as the updrafts are close to their level of neutral buoyancy, a larger proportion of the atmospheric layer has the same value of $\theta_{e}$.

The differences between $M^{+}, M_{\text {cor }}$, and $M_{\text {cld }}$ in the upper troposphere can be thought of in terms of the implicit time scales tied to the conditional averaging.
When averaging over convective cores, the key criterion is vertical velocity. In situations where overshoot or gravity waves occurs, the vertical velocity varies rapidly on time scale inversely proportional to the Brunt-Väisälä frequency (i.e., about $10 \mathrm{~min}$ ). In contrast, it may take several hours for a cloud to dissipate through mixing and precipitation. As a result, the averaging over clouds records air parcels for a longer period than the convective core. Similarly, as the radiative cooling rate in the upper troposphere is on the order of $1 \mathrm{~K} \mathrm{day}^{-1}$, while the fluctuations of temperature, measured for example as the difference of temperature between the updrafts and downdrafts in Fig. 1a, are on the order of 1 or $2 \mathrm{~K}$. This implies that the isentropic analysis includes a longer time scale: up to a couple of days. Qualitatively, such longer averaging window makes it easier to filter out fast oscillation such as gravity waves but also results in a larger number of air parcels being included. In the lower troposphere, air parcels rise rapidly, in a few minutes or so. This short ascent time is the primary factor that limits the contribution of any individual parcel to $\mathrm{M}^{+}, M_{\mathrm{cor}}$, and $M_{\text {cld }}$, so that the three mass fluxes and fractional areas are quite close.

Additional information on the nature of the overturning circulation can be extracted by determining the various thermodynamic properties of the rising and descending air. For a given variable $f$, we define its value in the mean ascent $f^{+}$and in the mean descent $f^{-}$as

$$
\begin{aligned}
& f^{+}(z)=\frac{1}{M^{+}} \int_{-\infty}^{\infty}\langle\rho w f\rangle H(\langle\rho w\rangle) d \theta_{e}^{\prime} \text { and } \\
& f^{-}(z)=\frac{1}{M^{-}} \int_{-\infty}^{\infty}\langle\rho w f\rangle H(-\langle\rho w\rangle) d \theta_{e}^{\prime} .
\end{aligned}
$$

The transport of a quantity $f$ by the mean ascent $F_{f}^{+}$ and by the mean descent $F_{f}^{-}$can then be written as

$$
\begin{aligned}
& F_{f}^{+}=M^{+}\left[f^{+}(z)-\bar{f}(z)\right] \quad \text { and } \\
& F_{f}^{-}=M^{-}\left[f^{-}(z)-\bar{f}(z)\right] .
\end{aligned}
$$

Note that in this definition, it is assumed that the upward or downward mass fluxes are compensated by an equal but opposite flux occurring at the horizontal-mean value $\bar{f}$.

We first apply these definitions to the moist static energy:

$$
H_{m}=C_{p} T+g Z+L_{v} q-L_{f} q_{i}
$$

where $C_{p}$ is heat capacity at constant pressure; $T$ is air temperature; $L_{v}$ and $L_{f}$ are, respectively, the latent heat 
of vaporization and fusion; and $q$ and $q_{i}$ are mass mixing ratios of water vapor and ice content, respectively. The mean updraft $H_{m}^{+}$, the mean downdraft $H_{m}^{-}$, and its horizontal-mean value $\overline{H_{m}}$ are shown in Fig. 6 a.

Below $10 \mathrm{~km}$, the mean ascent has a higher moist static energy than the mean descent, corresponding to an upward energy transport. However, above $10 \mathrm{~km}$, the moist static energy of the rising air $H_{m}^{+}$is less than that of the subsiding air $H_{m}^{-}$. This is a result of the convective overshooting, in which ascending air parcels rise above their level of neutral buoyancy then mix with environmental air with a higher value of the potential temperature before falling down. The definition of moist static energy [Eq. (19)] includes latent heat of fusion; thus, the presence of falling snow and ice causes a net upward energy flux, which may balance in part the downward convective energy transport in the upper troposphere.

The moist static energy in the mean downdraft is always very close to the horizontal mean, with $H_{m}^{-}-\overline{H_{m}}<0.5 \mathrm{~kJ} \mathrm{~kg}^{-1}$, which is consistent with the fact that the mean downdraft is in large part associated with subsiding air in the unsaturated environment. However, a closer examination indicates that the moist static energy in the mean downdraft is slightly higher than the horizontal-mean value everywhere except near the surface. This can be explained by the fact that a mixture of unsaturated environmental air and cloudy air has both higher moist static energy than the environmental air and higher density because of the reevaporation of condensate. Thus, one would expect such mixture to experience stronger downward motion, resulting in $H_{m}^{-}$to be slightly larger than the horizontal-mean moist static energy. While the effect here is small, such behavior indicates that the convective energy transport cannot be accurately represented by a simple Gaussian distribution around a mean atmospheric state.

In the upper troposphere, convective motions transport energy downward and act as a reverse heat engine that consumes kinetic energy to transport dense, cold air upward and lighter, warmer air downward. This can be verified by looking at the mean updraft $\left(B^{+}\right)$and mean downdraft $\left(B^{-}\right)$buoyancies shown in Fig. 6b. The buoyancy in the mean ascent is larger than the buoyancy in the mean descent between the surface and $10 \mathrm{~km}$, which corresponds to a net generation of kinetic energy by the convective motions. The opposite happens above $12 \mathrm{~km}$, with the mean ascent being denser than the mean descent, so that kinetic energy is being converted into geopotential and internal energy.

The two-stream approximation can be used to determine a bulk entrainment and detrainment rates associated with the mean updraft. It is assumed that the mass flux in the mean updraft increases or decreases with

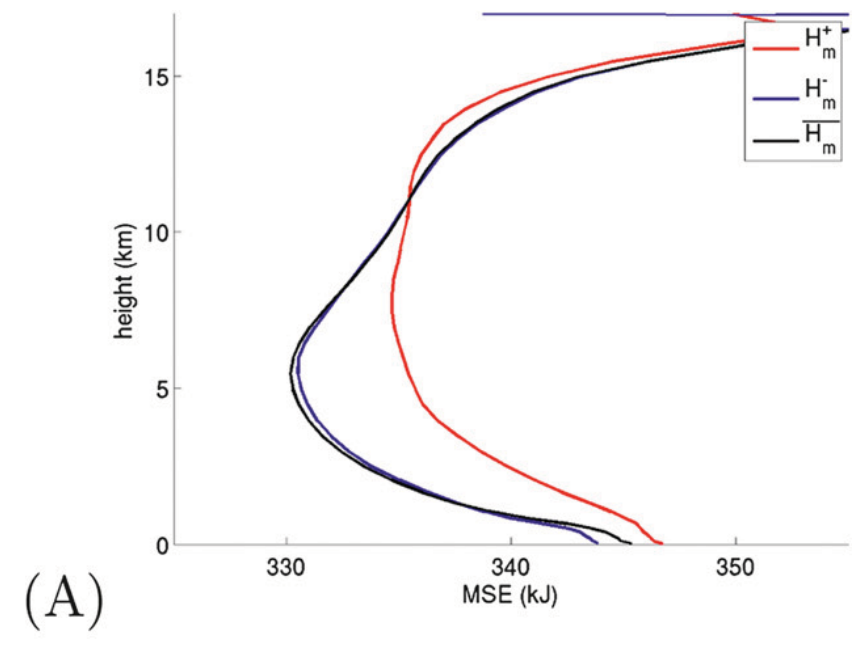

(B)
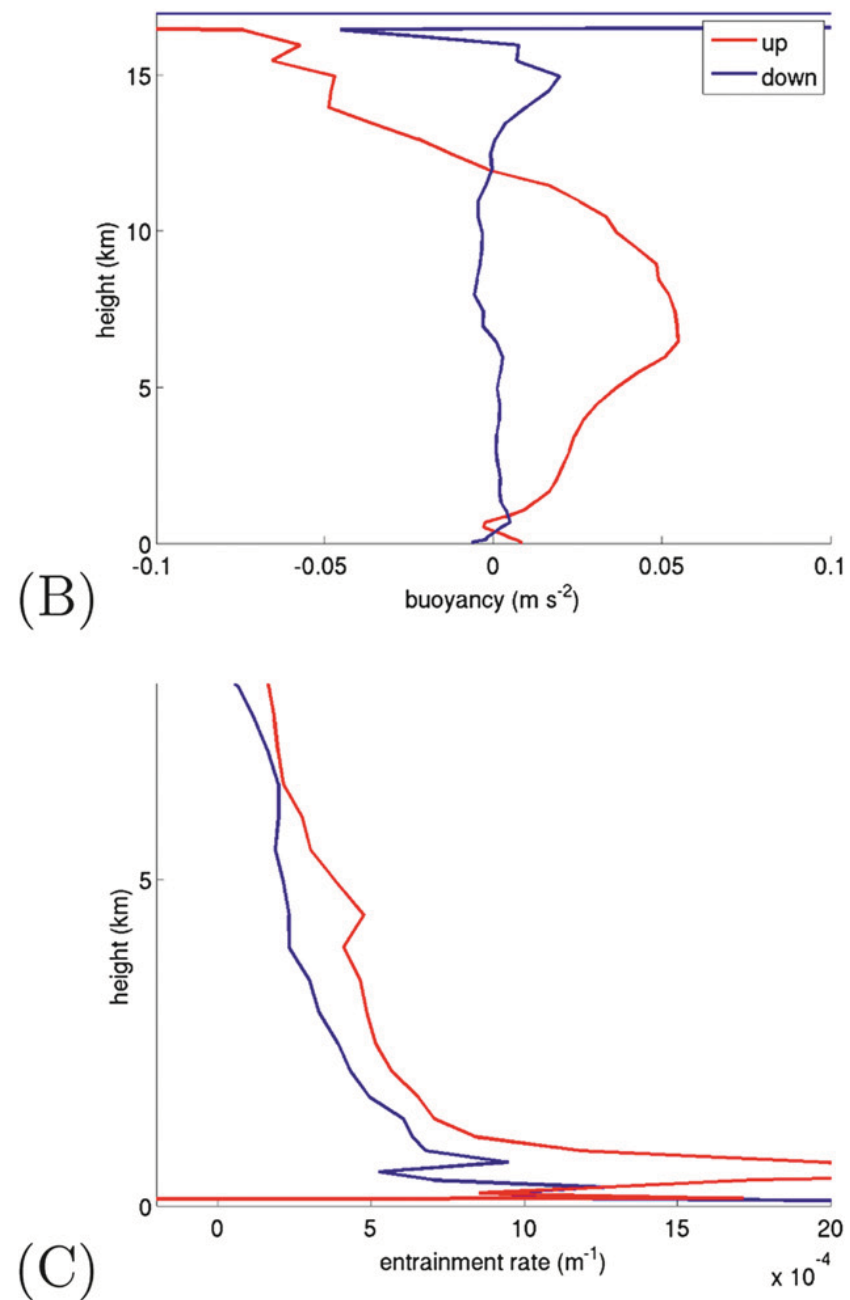

FIG. 6. Results from the two-stream diagnostic for the convection. (a) Moist static energy (kJ) in the updraft $H_{m}^{+}$, in the downdraft $H_{m}^{-}$, and horizontal-mean value $\overline{H_{m}}$. (b) Buoyancy $\left(\mathrm{m} \mathrm{s}^{-2}\right)$ in the updraft $B^{+}$and in the downdraft $B^{-}$. (c) Entrainment and detrainment rates $\left(\mathrm{m}^{-1}\right)$ based on Eqs. (22) and (23). 
height depending on the balance between entrainment and detrainment. At the same time, moist static energy in the updraft is diluted by the entrainment of environmental air. This means that $\mathrm{M}^{+}$and $H_{m}^{+}$in the mean updraft are governed by the following equations:

$$
\begin{aligned}
\frac{d M^{+}}{d z} & =(E-D) M^{+} \text {and } \\
\frac{d\left(M^{+} H_{m}^{+}\right)}{d z} & =E M^{+} \overline{H_{m}}-D M^{+} H_{m}^{+}+L_{f} P_{\text {ice }}
\end{aligned}
$$

where $E$ and $D$ are the fractional entrainment and detrainment rates $\left(\mathrm{m}^{-1}\right)$, respectively; $L_{f}$ is the latent heat of fusion; and $P_{\text {ice }}$ is the rate at which ice is removed by precipitation. If the vertical profiles of $\mathrm{M}^{+}$and $H_{m}^{+}$are known, the above equations can be solved for entrainment and detrainment rates:

$$
\begin{aligned}
E & =\frac{L_{f} P_{\text {ice }} / M^{+}-d H_{m}^{+} / d z}{H_{m}^{+}-\overline{H_{m}}} \text { and } \\
D & =E-\frac{1}{M^{+}} \frac{d M^{+}}{d z}
\end{aligned}
$$

Note that Eq. (22) may yield negative values for the entrainment rate if the moist static energy in the mean updraft increases with height, which can happen in the upper troposphere. This is a limitation of the simplified model [Eqs. (20) and (21)], which considers a single rising plume to account for all convective motions. As can be deduced from Fig. 1, detrainment in the upper troposphere is associated preferentially with air parcels that have lower values of $\theta_{e}$ than the mean updraft (and hence a lower moist static energy). Nevertheless, the results from this simple model appear reasonable in the lower $8-10 \mathrm{~km}$ of the atmosphere and are shown in Fig. $6 c$. The detrainment rate peaks at the cloud base, decreases with height, and is always higher than the entrainment rate, consistent with the mean updraft mass flux decreasing with height. The entrainment rate varies from $6 \times 10^{-4} \mathrm{~m}^{-1}$ right above the cloud base to about $2 \times 10^{-4} \mathrm{~m}^{-1}$ at $8 \mathrm{~km}$. This indicates that while there is vigorous entrainment in the lower troposphere, it becomes less important from the middle troposphere.

As discussed in de Rooy et al. (2013), such bulk entrainment and detrainment rates are sensitive to details in how the bulk model is formulated. For example, Siebesma and Cuipers (1995) and Siebesma et al. (2003) derive the entrainment and detrainment rates from the transport of total water and liquid water potential temperature, while Romps (2010) uses a purity tracer. Unfortunately, these different choices can directly affect the numerical values for the entrainment and detrainment rates. Our analysis indicates that the choice in the definition of the bulk mass transport has also a large impact for the retrieval of $E$ and $D$. Indeed, had we used the mass flux in clouds to the convective core as it is commonly done, we would have found very large value of the entrainment rate in the upper troposphere where these mass fluxes increase with height. Such large values, however, are an artifact of the fact that both $M_{\text {cor }}$ and $M_{\text {cld }}$ include a significant contribution from gravity waves in their estimate of the mass flux. By contrast, the use of the isentropic analysis to compute $M^{+}$offers a robust way to estimate the convective mass transport that excludes oscillatory motions such as gravity waves. Any bulk approach, including the two-stream approximation outlined here, remains limited by the fact that it attempts to aggregate a complex flow into a single mass transport. A better understanding of entrainment and detrainment processes should describe how they vary for different parcels, which could be done based on a conditional average version of Eq. (12) or the local definition of entrainment introduced by Romps (2010).

\section{Discussion}

In this paper, a new method of analysis for convective motions in high-resolution simulations has been proposed. Our approach relies on conditional averaging of the various properties of air parcels in terms of both the height and the equivalent potential temperature. This averaging procedure reduces a four-dimensional dataset into a two-dimensional distribution by compressing fluctuations in the two horizontal coordinates and time into a single thermodynamic coordinate $\left(\theta_{e}\right)$. While other studies such as Yano et al. (2004) and Pauluis and Garner (2006) have analyzed the behavior of convection in terms of the vertical velocity distribution, a conditional averaging based on equivalent potential temperature has the advantage of preserving the separation between the ascent of warm, moist air and subsidence of colder, drier air, which are the fundamental aspects of moist convection. Furthermore, while the equivalent potential temperature of an air parcel can be affected by a wide range of processes, such as radiation, evaporation, and mixing, it is conserved for reversible adiabatic transformation. As defined, a conditional averaging on $\theta_{e}$ can be viewed as isolating the irreversible convective overturning by filtering out fast, reversible oscillatory motions such as gravity waves.

The conditional averaging has been first used here to extract a vertical mass flux and to compute an isentropic streamfunction. Analysis of the streamfunction identifies the convective overturning as a combination of 
ascents of high energy air parcels and descents of air with much lower energy, shows the role of entrainment in reducing the equivalent potential temperature of the rising air parcels in the lower troposphere, and indicates the presence of convective overshoot in the tropopause region. There is a strong asymmetry between updrafts and downdrafts, with the former occupying a small area but occurring at fairly large vertical velocity, and the latter being associated with slow subsidence over the large portion of the domain. Very strong updrafts, with vertical velocities reaching $30 \mathrm{~m} \mathrm{~s}^{-1}$ corresponding to a rare occurrence of almost undiluted air parcels ascending from the boundary layer, were also observed. The scarcity of undiluted updrafts is in agreement with the recent findings of Romps and Kuang (2010). Other important properties of rising and subsiding parcels, such as temperature, humidity, and buoyancy, can also be systematically studied with the isentropic-averaging approach.

Diabatic tendencies can be computed from the continuity equation expressed in $z-\theta_{e}$ coordinates without the need to compute them explicitly within the model. When applied to the radiative-convective equilibrium simulations, our analysis shows that entrainment reduces the equivalent potential temperature in the updrafts (especially in the lower troposphere), while detrainment increases the equivalent potential temperature in subsiding air parcels. Near the freezing level, a slight increase in the equivalent potential temperature of the updrafts was also found, which can be attributed to the freezing of condensed water. Empirical entrainment rate can also be determined as a function of both height and the equivalent potential temperature. This analysis confirms the presence of significant entrainment in the lower troposphere, while the rare occurrence of updrafts with high values of $\theta_{e}$ corresponds to air parcels that have experienced little to no entrainment.

The choice of the variable used in the conditional averaging (1) is somewhat arbitrary. We choose the equivalent potential temperature $\theta_{e}$ albeit without including a contribution from the ice phase or precipitation (i.e., the rain, snow, and graupel species from the microphysics scheme). Our decision to exclude the precipitation water is motivated by our desire to avoid rapid changes in $\theta_{e}$ that would occur when precipitation falls through an air parcel. Different choices could have been made, such as moist entropy, a formulation of the equivalent potential temperature that explicitly includes an ice phases, or moist static energy. It is, however, not possible to obtain a thermodynamic variable that is exactly conserved for an ascending parcel that loses condensed water or ice. In practice, one would like to use a variable that is approximately conserved for undiluted ascent, but exact conservation is not necessary (nor achievable for actual air parcels) to separate updrafts and downdrafts.

While one of the main motivations for the isentropic averaging is to obtain a statistical description of convective motions by separating air parcels in terms of their equivalent potential temperature, it is also possible to further synthesize the information in terms of a twostream approximation. This method defines a mean ascent and a mean descent based on the isentropic streamfunction. In doing so, one can describe a convective mass transport as well as the mean updraft and downdraft properties. Results from the two-stream approximation have been compared with standard definitions of the mass transport inside clouds and inside convective cores. It is shown that the isentropic analysis leads to systematically lower values for the mass transport due to the fact that the isentropic analysis filters out gravity waves (as long as these correspond to reversible oscillations of air parcels around their level of neutral buoyancy), while other averaging techniques tend to include additional contributions from gravity waves.

The technique proposed here can be regarded as an equivalent of the analysis of the meridional circulation in isentropic coordinates (Dutton 1976; Johnson 1989; Held and Schneider 1999; Pauluis et al. 2008, 2010) applied to the vertical transport by convection. As convective motions act to continuously stretch and fold isentropic surfaces, their geometry can be very complex. The isentropic averaging can be viewed as a useful mathematical tool to disentangle this complex geometry. The isentropic averaging also serves as a quasiLagrangian coordinate system that filters out fast reversible oscillations and captures the core convective processes associated with high-entropy updrafts balanced by slow subsidence of low-entropy air. The approach presented here is well suited for analysis of simulated convection. In addition to reduction of complex four-dimensional datasets into more manageable two-dimensional distributions, the isentropic analysis offers the possibility of recovering the diabatic tendencies without requiring detailed knowledge of the numerical models. This can be advantageous as one tries to diagnose the convective transport in increasingly complex numerical models in which the diabatic tendencies result from an array of physical parameterizations, including turbulent closure, microphysics, and radiative transfer. While direct computation of the isentropic streamfunction requires a significant amount of data, it might be possible to approximate it accurately on the basis of a statistical approximation, as it can be done using the statistical transformed Eulerian-mean circulation to reconstruct the global isentropic circulation (Pauluis 
et al. 2011). Hence, the isentropic streamfunction could potentially be used as an intermediary diagnostic for comparison between high-resolution cloud-resolving models and single-column models.

Finally, a potential application of the isentropic averaging lies in the reconstruction of the transformations that various air parcels undergo as they are being transported within the convective systems. In fact, the isentropic averaging can recover not only the convective mass transport, but also the various thermodynamic properties. It is thus possible to use this information to at least approximate the thermodynamic evolution of air parcels. We plan to investigate such technique in an upcoming paper.

Acknowledgments. We are thankful to the two anonymous reviewers and the editor for their constructive comments on the manuscript. Olivier Pauluis is supported by the NSF under Grant AGS-0944058 and by the New York University Abu Dhabi Research Institute. Agnieszka Mrowiec's contribution to this research was supported by the DOE Office of Science, Office of Biological and Environmental Research, through Contract DE-PS02-09ER09-01 within the scope of the FASTER Project.

\section{APPENDIX}

\section{Isentropic Average of the Continuity Equation}

We derive here Eq. (9), which amounts to a statement of conservation of mass after isentropic averaging.

First, we define the mass $M$ of air below a level $z$ and potential temperature $\theta_{e}$ as

$$
M\left(z, \theta_{e 0}, t\right)=\frac{1}{L_{x} L_{y}} \int_{0}^{z} \int_{0}^{L_{y}} \int_{0}^{L_{x}} \rho H\left(\theta_{e 0}-\theta_{e}\right) d x d y d z,
$$

where $H$ is the Heaviside function. The time derivative of $M$ is given by

$$
\frac{\partial M}{\partial t}=\frac{1}{L_{x} L_{y}} \int_{0}^{z} \int_{0}^{L_{y}} \int_{0}^{L_{x}} \frac{\partial \rho}{\partial t} H\left(\theta_{e 0}-\theta_{e}\right)-\rho \delta\left(\theta_{e 0}-\theta_{e}\right) \frac{\partial \theta_{e}}{\partial t} d x d y d z,
$$

where we use the fact that $(\partial / \partial t) H\left(\theta_{e 0}-\theta_{e}\right)=$ $-\delta\left(\theta_{e 0}-\theta_{e}\right)\left(\partial \theta_{e} / \partial t\right)$. We consider a general form for the $\theta_{e}$ equation to be

$$
\frac{\partial \theta_{e}}{\partial t}+\mathbf{V} \cdot \nabla \theta_{e}=\dot{\theta}_{e}
$$

with $\mathbf{V}$ the three-dimensional vector field and $\dot{\theta}_{e}$ the rate of change of $\theta_{e}$ resulting from the various physical process on the parcel. Using this equation as well as the continuity equation, we can now write

$$
\begin{aligned}
\frac{\partial M}{\partial t} & =\frac{1}{L_{x} L_{y}} \int_{0}^{L_{y}} \int_{0}^{L_{x}} \int_{0}^{z}-H\left(\theta_{e 0}-\theta_{e}\right) \nabla \cdot(\rho \mathbf{V})+\delta\left(\theta_{e 0}-\theta_{e}\right) \rho \mathbf{V} \cdot \nabla \theta_{e}-\delta\left(\theta_{e 0}-\theta_{e}\right) \rho \dot{\theta}_{e} d z d x d y \\
& =\frac{1}{L_{x} L_{y}} \int_{0}^{L_{y}} \int_{0}^{L_{x}} \int_{0}^{z}-\nabla \cdot\left[\rho \mathbf{V} H\left(\theta_{e 0}-\theta_{e}\right)\right]-\delta\left(\theta_{e 0}-\theta_{e}\right) \rho \dot{\theta}_{e} d z d x d y \\
& =-\frac{1}{L_{x} L_{y}} \int_{0}^{L_{y}} \int_{0}^{L_{x}} \rho w H\left(\theta_{e 0}-\theta_{e}\right) d x d y-\frac{1}{L_{x} L_{y}} \int_{0}^{L_{y}} \int_{0}^{L_{x}} \int_{0}^{z} \delta\left(\theta_{e 0}-\theta_{e}\right) \rho \dot{\theta}_{e} d z d x d y .
\end{aligned}
$$

In deriving Eq. (A3), we have used the fact that

$$
\delta\left(\theta_{e 0}-\theta_{e}\right) \rho \mathbf{V} \cdot \nabla \theta_{e}=-\rho \mathbf{V} \cdot \nabla\left[H\left(\theta_{e 0}-\theta_{e}\right)\right] .
$$

While the isentropic integral defined in Eq. (1) includes a time averaging, it can be extended to define the instantaneous isentropic integral of a quantity $f$ as

$$
\langle f\rangle_{*}\left(z, \theta_{e 0}, t\right)=\frac{1}{L_{x} L_{y}} \int_{0}^{L_{y}} \int_{0}^{L_{x}} f(x, y, z, t) \delta\left[\theta_{e 0}-\theta_{e}(x, y, z, t)\right] d x d y .
$$


Under this notation, the isentropic integral $\langle\rho\rangle_{*}$ is given by

$$
\langle\rho\rangle_{*}\left(z, \theta_{e 0}, t\right)=\frac{\partial^{2} M}{\partial z \partial \theta_{e 0}} .
$$

If we now apply the second-order derivative $\partial^{2} / \partial \theta_{e 0} \partial z$ to Eq. (A3), we obtain

$$
\frac{\partial}{\partial t}\langle\rho\rangle_{*}+\frac{\partial}{\partial z}\langle\rho w\rangle_{*}+\frac{\partial}{\partial \theta_{e 0}}\left\langle\rho \dot{\theta}_{e}\right\rangle_{*}=0 .
$$

This amounts to conservation of mass in the $z-\theta_{e}$ coordinates. We can take the time average of Eq. (A5), so that the instantaneous integral $\langle\cdot\rangle_{*}$ is replaced by the time-average counterpart $\langle\cdot\rangle$. For a statistically steady system, the first term on the left-hand side vanishes and we are left with Eq. (9):

$$
\frac{\partial}{\partial z}\langle\rho w\rangle+\frac{\partial}{\partial \theta_{e 0}}\left\langle\rho \dot{\theta}_{e}\right\rangle=0
$$

\section{REFERENCES}

Andrews, D. G., and M. E. McIntyre, 1978: An exact theory of nonlinear waves on a Lagrangian-mean flow. J. Fluid Mech., 89, 609-646.

—, J. R. Holton, and C. B. Leovy, 1987: Middle Atmospheric Dynamics. 1st ed. International Geophysics Series, Vol. 40, Academic Press, 489 pp.

Bjerkness, J., 1938: Saturated-adiabatic ascent of air through dryadiabatically descending environment. Quart. J. Roy. Meteor. Soc., 64, 325-330.

Browning, K., and F. Ludlam, 1962: Airflow in convective storms. Quart. J. Roy. Meteor. Soc., 88, 117-137.

de Rooy, W., and Coauthors, 2013: Entrainment and detrainment in cumulus convection: An overview. Quart. J. Roy. Meteor. Soc., 139, 1-19.

Dutton, J., 1976: The Ceaseless Wind. McGraw-Hill, 579 pp.

Emanuel, K. A., 1994: Atmospheric Convection. Oxford University Press, $580 \mathrm{pp}$.

Green, J., F. Ludlam, and J. McIlveen, 1966: Isentropic relativeflow analysis and the parcel theory. Quart. J. Roy. Meteor. Soc., 92, 210-219.

Harrold, T., 1973: Mechanisms influencing the distribution of precipitation within baroclinic structure. Quart. J. Roy. Meteor. Soc., 99, 232-251.

Held, I. M., and T. Schneider, 1999: The surface branch of the zonally averaged mass transport circulation in the troposphere. J. Atmos. Sci., 56, 1688-1697.

Johnson, D. R., 1989: The forcing and maintenance of global monsoonal circulations: An isentropic analysis. Advances in Geophysics, Vol. 31, Academic Press, 43-316.
Khairoutdinov, M. F., and D. A. Randall, 2003: Cloud resolving modeling of the ARM summer 1997 IOP: Model formulation, results, uncertainties, and sensitivities. J. Atmos. Sci., 60, 607625.

Kuang, Z., and C. Bretherton, 2006: A mass-flux scheme view of a high-resolution simulation of a transition from shallow to deep cumulus convection. J. Atmos. Sci., 63, 1895-1909.

Ludlam, F., 1963: Severe local storms: A review. Severe Local Storms, Meteor. Monogr., No. 27, Amer. Meteor. Soc., 1-30.

McIntyre, M. E., 1980: An introduction to the generalized Lagrangian-mean description of wave, mean-flow interaction. Pure Appl. Geophys., 118, 152-176.

Merrill, R. T., and C. S. Velden, 1996: A three-dimensional analysis of the outflow layer of Supertyphoon Flo (1990). Mon. Wea. Rev., 124, 47-63.

Molinari, J., S. Skubis, and D. Vollaro, 1995: External influences on hurricane intensity. Part III: Potential vorticity structure. J. Atmos. Sci., 52, 3593-3606.

—, D. Knight, M. Dickinson, D. Vollaro, and S. Skubis, 1997: Potential vorticity, easterly waves, and eastern Pacific tropical cyclogenesis. Mon. Wea. Rev., 125, 2699-2708.

Pauluis, O., 2008: Thermodynamic consistency of the anelastic approximation for a moist atmosphere. J. Atmos. Sci., 65, 2719-2729.

— , and S. Garner, 2006: Sensitivity of radiative convective equilibrium simulations to horizontal resolution. J. Atmos. Sci., 63, 1910-1923.

— A. Czaja, and R. Korty, 2008: The global atmospheric circulation on moist isentropes. Science, 321, 1075-1078, doi:10.1126/ science. 1159649.

,$- \ldots$, and -2010 : The global atmospheric circulation in moist isentropic coordinates. J. Climate, 23, 3077-3093.

_ - T. Shaw, and F. Laliberte, 2011: A statistical generalization of the transformed Eulerian-mean circulation for an arbitrary vertical coordinate system. J. Atmos. Sci., 68, 1766-1783.

Romps, D. M., 2010: A direct measure of entrainment. J. Atmos. Sci., 67, 1908-1927.

— and Z. Kuang, 2010: Do undiluted convective plumes exist in the upper tropical troposphere? J. Atmos. Sci., 67, 468-484.

Rossby, C.-G., 1937: Isentropic analysis. Bull. Amer. Meteor. Soc., 18, 201-209.

Schubert, W. H., and B. T. Alworth, 1987: Evolution of potential vorticity in tropical cyclones. Quart. J. Roy. Meteor. Soc., 113, $147-162$.

Siebesma, A. P., and J. Cuipers, 1995: Evaluation of parametric assumptions for shallow cumulus convection. J. Atmos. Sci., 52, 650-667.

—, and Coauthors, 2003: A large eddy simulation intercomparison study of shallow cumulus convection. J. Atmos. Sci., 60, 1201-1219.

Stevens, B., 2005: Atmospheric moist convection. Annu. Rev. Earth Planet. Sci., 33, 605-643.

$\mathrm{Xu}$, K.-M., and K. A. Emanuel, 1989: Is the tropical atmosphere conditionally unstable? Mon. Wea. Rev., 117, 1471-1479.

Yano, J.-I., F. Guichard, J.-P. Lafore, J.-L. Redelperger, and P. Bechtold, 2004: Estimations of mass fluxes for cumulus parameterizations from high-resolution spatial data. J. Atmos. Sci., 61, 829-842. 